\title{
ON THE ROOTS OF SPECTRAL OPERATORS
}

\section{CONSTANTIN APOSTOL}

Introduction. Let $X$ be a Banach space and $T$ a linear bounded operator acting in $X$. It is known that if $T$ is invertible and $T^{n}$ is a spectral operator for some natural number $n$, then $T$ is spectral (see [9]).

Our principal result (Theorem 2.1) is a sufficient (and evidently necessary) condition for $T$ to be a spectral operator when $T^{n}$ is spectral.

1. Throughout we shall denote by $X$ a Banach space and by $\&(X)$ the algebra of all linear bounded operators acting in $X . N$ resp. $\Lambda$ will be the set of all natural resp. complex numbers. The field of all Borel subsets of $\Lambda$ will be denoted by $B$. For $n \in N, \sigma \in \Theta$ we shall write $\sigma^{1 / n}$ for the set $\left\{\lambda: \lambda \in \Lambda, \lambda^{n} \in \sigma\right\}$. For any $0 \leqq r$ we shall denote $\Lambda_{r}=\{\lambda: \lambda \in \Lambda, r<|\lambda|\}$.

Lemma 1.1. Let $\sigma, \tau \in ß$ and $n, m \in N$ be two relatively prime numbers. If we have $|\arg \lambda-\arg \mu|<2 \pi / m n$ for any $\lambda, \mu \in \sigma \cup_{\tau}, \lambda \mu \neq 0$, then the equality $\left(\sigma^{m}\right)^{1 / m} \cap\left(\tau^{n}\right)^{1 / n}=\sigma \cap \tau$ holds.

Proof. Evidently we have $\sigma \cap \tau \subset\left(\sigma^{m}\right)^{1 / m} \cap\left(\tau^{n}\right)^{1 / n}$. Let now $\zeta \in\left(\sigma^{m}\right)^{1 / m} \cap\left(\tau^{n}\right)^{1 / n}$. It follows that there exist $\lambda \in \sigma, \mu \in \tau$ such that $\lambda^{m}=\zeta^{m}, \mu^{n}=\zeta^{n}$. If $\zeta=0$ then $\zeta=\lambda=\mu$ thus $\lambda \in \sigma \cap \tau$. Let us suppose that $\zeta \neq 0$. Then we have $|\zeta|=|\lambda|=|\mu|$ and there exist two integers $k, r$ such that $|k|<m,|r|<n$ and $\arg \zeta-\arg \lambda=2 k \pi / m, \arg \zeta-\arg \mu$ $=2 r \pi / n$.

It follows that $|\arg \lambda-\arg \mu|=(2 \pi / m n)|k n-r m|<2 \pi / m n$; thus $|k n-r m|=0$. Since $m$ and $n$ are relatively prime the last inequality implies $k=r=0$ thus $\zeta=\lambda=\mu \in \sigma \cap \tau$ which proves our lemma.

Let us now suppose that $T^{m}$ and $T^{n}$ are spectral operators, $m, n \in N$, with the spectral measures $F$ and respectively $G$.

For any $0 \leqq r$ the subspace $X_{r}=F\left(\Lambda_{r} m\right) X$ is ultra-invariant for $T$ (that is invariant for all operators commuting with $T$ ) as a consequence of the properties of $F$. For any $0<r$ the operator $T \mid X_{r}$ is invertible and since $\left(T \mid X_{r}\right)^{m}=T^{m} \mid X_{r}$ is spectral, it follows that $T \mid X_{r}$ is spectral [9]. Let $E_{r}$ be the spectral measure of $T \mid X_{r}$.

Lemma 1.2. Let $m, n \in N$ be two relatively prime numbers such that $T^{m}$ and $T^{n}$ are spectral operators and $0 \leqq \alpha<\beta \leqq 2 \pi, \beta-\alpha \leqq 2 \pi / m n$.

Received by the editors March 25, 1967. 
If $A=\{\lambda: \lambda \in \Lambda \backslash\{0\}, \alpha \leqq \arg \lambda<\beta\}$, then the map $E_{A}$, defined by the equation

$$
E_{A}(\sigma)=F\left((\sigma \cap A)^{m}\right) G\left((\sigma \cap A)^{n}\right),
$$

is a homomorphic map of $B$ into a Boolean algebra of projection operators in $X$ with the identity $E_{A}(A) . E_{A}(\sigma)$ is strongly countably additive as a function of $\sigma$.

Proof. It is easily seen that we have $F\left(\Lambda_{r} m\right)=G\left(\Lambda_{r} n\right)$ for any $0 \leqq r$. Now by Theorem 2.4.1 of [3], the operators $T^{m}\left|X_{r}, T^{n}\right| X_{r}$ are spectral since $X_{r}$ is an ultra-invariant subspace for $T$ and henceforth for $T^{m}$ and $T^{n}$. Also by the same theorem the spectral measures of these operators are $F \mid X_{r}$ and $G \mid X_{r}$.

Now if $0<r$, the operator $T \mid X_{r}$ is spectral, and by Lemma 6 [5], the spectral measure of $\left(T \mid X_{r}\right)^{m},\left(T \mid X_{r}\right)^{n}$ is $\sigma \rightarrow E_{r}\left(\sigma^{1 / m}\right) \mid X_{r}$ and respectively $\sigma \rightarrow E_{r}\left(\sigma^{1 / n}\right) \mid X_{r}$.

Using the equality $T^{k} \mid X_{r}=\left(T \mid X_{r}\right)^{k}$ for any $k \in N$ and the uniqueness of the spectral measure of a spectral operator we obtain for any $\sigma, \tau \in B$

$$
\begin{aligned}
E_{r}\left(\left[(\sigma \cap A)^{m}\right]^{1 / m}\right) F\left(\Lambda_{\tau^{m}}\right) & =F\left((\sigma \cap A)^{m}\right) F\left(\Lambda_{r} m\right), \\
E_{r}\left(\left[(\tau \cap A)^{n}\right]^{1 / n}\right) F\left(\Lambda_{r} m\right) & =G\left((\tau \cap A)^{n}\right) F\left(\Lambda_{r} m\right) .
\end{aligned}
$$

Now by Lemma 1.1 it follows that

$$
E_{r}(\sigma \cap \tau \cap A) F\left(\Lambda_{r^{m}}\right)=F\left((\sigma \cap A)^{m}\right) G\left((\tau \cap A)^{n}\right) F\left(\Lambda_{r^{m}}\right),
$$

thus

$$
E_{A}(\sigma)=\lim _{r \rightarrow 0} E_{r}(\sigma \cap A) F\left(\Lambda_{r^{m}}\right) .
$$

Evidently $E_{A}$ is a homomorphism and $E_{A}(A)$ is the identity of the range of $E_{A}$.

Now if $\sigma=\bigcup_{\mathbf{k}=0}^{\infty} \sigma_{k}, \sigma_{k} \cap \sigma_{j}=\varnothing, k \neq j$, we have for any $x \in X$

$$
\begin{aligned}
E_{A}(\sigma) x & =\sum_{k=0}^{\infty} F\left(\left(\sigma_{k} \cap A\right)^{m}\right) G\left((\sigma \cap A)^{n}\right) x \\
& =\sum_{k=0}^{\infty} F\left(\left(\sigma_{k} \cap A\right)^{m}\right) G\left(\left(\sigma_{k} \cap A\right)^{n}\right) x=\sum_{k=0}^{\infty} E_{A}\left(\sigma_{k}\right) x
\end{aligned}
$$

which proves the countable additivity of $E_{A}$.

2. Further $m$ and $n$ will be two fixed relatively prime numbers, $m, n \in N, 2 \leqq m<n$. 
For any $k=0,1, \cdots, m n$, we shall denote $A_{k}=\{\lambda: \lambda \in \Lambda \backslash\{0\}, 2 k \pi /(n m+1) \leqq \arg \lambda<(2 k+1) \pi /(n m+1)\}$.

TheOREM 2.1. If $T^{m}$ and $T^{n}$ are spectral operators then $T$ is a spectral operator.

Proof. Let $Y=F(\{0\}) X, X_{k}=E_{A_{k}}\left(A_{k}\right) X, k=0,1, \cdots, m n$. We have:

$$
\begin{aligned}
F(\{0\}) E_{A_{k}}\left(A_{k}\right) & =\lim _{r \rightarrow 0} F(\{0\}) E_{r}\left(A_{k}\right) F\left(\Lambda_{r} m\right)=0, \\
E_{A_{k}}\left(A_{k}\right) E_{A_{j}}\left(A_{j}\right) & =\lim _{r \rightarrow 0} E_{r}\left(A_{k}\right) E_{r}\left(A_{j}\right) F\left(\Lambda_{r} m\right)=0, \quad k \neq j,
\end{aligned}
$$

and

$$
\sum_{k=0}^{m n} E_{A_{k}}\left(A_{k}\right)=\lim _{r \rightarrow 0} \sum_{k=0}^{m n} E_{r}\left(A_{k}\right) F\left(\Lambda_{r} m\right)=\lim _{r \rightarrow 0} F\left(\Lambda_{r} m\right)=F(\Lambda \backslash\{0\}) .
$$

It follows that $X$ is the direct sum

$$
X=Y \oplus \bigoplus_{k=0}^{m n} X_{k}, \quad T=(T \mid Y) \oplus \bigoplus_{k=0}^{m n}\left(T \mid X_{k}\right) .
$$

We have $\sigma(T \mid Y) \subset\{0\}$; thus $T \mid Y$ is a spectral operator. Also $E_{A_{k}} \mid X_{k}$ is a resolution of the identity for $T \mid X_{k}$. Indeed, if we denote $E_{k}=E_{A_{k}} \mid X_{k}$, we have evidently $E_{k}(\sigma)\left(T \mid X_{k}\right)=\left(T \mid X_{k}\right) E_{k}(\sigma)$ and also $\sigma\left(\left(T \mid X_{k}\right)^{m} \mid E_{k}(\sigma) X_{k}\right) \subset\left(\bar{\sigma} \cap \bar{A}_{k}\right)^{m}, \sigma\left(\left(T \mid X_{k}\right)^{n} \mid E_{k}(\sigma) X_{k}\right) \subset\left(\bar{\sigma} \cap \bar{A}_{k}\right)^{n}$.

By the spectral mapping theorem [4] and Lemma 1.1 we obtain $\sigma\left(\left(T \mid X_{k}\right) \mid E_{k}(\sigma) X_{k}\right) \subset \bar{\sigma}$.

Using Lemma 1.2 it results that $T \mid X_{k}$ is a spectral operator (see [4]). Thus $T$ is a spectral operator as the direct sum of spectral operators.

Theorem 2.2. In the hypothesis of Theorem 2.1, if $T^{m}$ is of scalar type then $T$ is a spectral operator of finite type. Let $S$ be its scalar part and $Q$ its nilpotent part. Then we have $S Q=Q S=0, Q^{m}=0$.

Proof. By the preceding theorem we have $T=S+Q$ where $S$ is of scalar type and $Q$ a generalised nilpotent. Let $E$ be the spectral measure of $T$. Since $T^{m}$ is scalar we have $T^{m}=S^{m}$, and $T \mid E\left(\Lambda_{r}\right) X$ is also scalar [9]. From the equality $T F\left(\Lambda_{r}\right)=S F\left(\Lambda_{r}\right)+Q F\left(\Lambda_{r}\right)$ it results that $Q F\left(\Lambda_{r}\right)=0$ is the radical part of $T \mid F\left(\Lambda_{r}\right)$. Consequently $Q=Q F(\{0\})$.

It follows that $S Q=Q S=S F(\{0\}) Q=0, S^{m}=T^{m}=S^{m}+Q^{m}$. Thus $Q^{m}=0$. 
Corollary 2.3 (of Theorem 2.2). If 0 is not an eigenvalue of $T$ then $T$ is a scalar operator.

Proof. By Theorem 2.2, $S Q=0$, and since 0 is not an eigenvalue of $T$ and $S^{m} Q=T^{m} Q=0$, it follows that $Q=0, T=S$.

\section{BIBLIOGRAPHY}

1. C. Apostol, Propriétés de certains opérateurs bornés des espaces de Hilbert, Rev. Math. Pures Appl. 10 (1965), 643-644.

2. - Propriétés de certains opérateurs bornés des espaces de Hilbert. II, Rev. Math. Pures Appl. 12 (1967), 759-762.

3. R. Dowson, Restriction of spectral operators, Proc London Math. Soc. 15 (1965), $437-457$.

4. N. Dunford and J. T. Schwartz, Linear operators, Part I, Interscience, New York, 1958.

5. - Spectral operators, Pacific J. Math. 4 (1954), 321-354.

6. S. Foguel, Sums and products of commuting spectral operators, Ark. Mat. 3(1958), 449-461.

7. S. Kurepa, On n-th roots of normal operators, Math. Z. 78 (1962), 285-292.

8. C. R. Putnam, On square roots of normal operators, Proc. Amer. Math. Soc. 8 (1957), 768-769.

9. J. G. Stampfli, Roots of scalar operators, Proc. Amer. Math. Soc. 13 (1962), 796-798.

Académie de la Republique Société de Roumaine, Bucarest 